\title{
Nonparallel local spatial stability analysis of pipe entrance swirling flows
}

\author{
M. A. Herrada, M. Pérez-Saborid, and A. Barrero \\ Escuela Superior de Ingenieros, Universidad de Sevilla, 41092 Sevilla, Spain
}

(Received 29 October 2003; accepted 9 March 2004; published online 12 May 2004)

\begin{abstract}
A spatial local viscous stability analysis of a swirling flow developing in a cylindrical pipe has been carried out numerically. Even at moderately low swirl strengths, we have found the existence of centrifugal modes in addition to the shear ones found in previous stability analysis of nonswirling flows developing in pipes. It is found that these centrifugal instabilities develop at Reynolds numbers that are much lower than those required for the growing of the shear instability. Moreover, the extent of the region where centrifugal instabilities appear is much larger than that where the shear layer instability grows. We have found from the analysis that the most unstable mode was the counter-rotating one $(n=-1)$. The critical Reynolds number for which linear analysis predicts the growth of the convective instabilities is for the centrifugal modes one hundred times smaller than for the shear layer ones. (C) 2004 American Institute of Physics. [DOI: 10.1063/1.1728158]
\end{abstract}

\section{INTRODUCTION}

The effect of the swirl strength on the stability of developing swirling flows in pipes has not been sufficiently investigated yet. It is well known that in the absence of swirl, the fully developed Poiseuille flow in a pipe is stable to infinitesimal axisymmetric and nonaxisymmetric disturbances under both temporal and spatial analyses. This fact motivated the study of the linear stability of the developing flow in the pipe entrance. ${ }^{1-5}$ Even though the latter analyses succeed in ascertaining regions of instability within the laminar entrance region, they yield critical Reynolds numbers for instability whose value differs from one study to another. The discrepancies between the existing analyses may be attributed to the calculation of the basic flow, to the different approaches (spatial or temporal) to the stability problem, and also to the different treatment of the nonparallel terms in the linearized equations. Even worse is the disagreement between the theoretical results and the experimental ones, ${ }^{6}$ the finite amplitude nature of the applied disturbances and possible bypass mechanisms were suggested as a possible explanation of these discrepancies. ${ }^{5}$

The linear stability results for the fully developed Poiseuille flow in pipes are modified significantly in the presence of swirl. In effect, the temporal stability analysis first carried out by Pedley ${ }^{7,8}$ showed that Poiseuille flow with superimposed solid body rotation becomes unstable to nonaxisymmetric disturbances, while it remains stable to axisymmetric ones. These results were later confirmed and extended by more complete temporal stability studies. ${ }^{9-12}$ It was found there that the most unstable disturbance corresponded to the mode with azimuthal wave number $n=-1$. Spatial stability analyses ${ }^{13,14}$ of the same basic flow have shown that the flow was convectively unstable under nonaxisymmetric perturbations; the most unstable mode being precisely the $n=-1$ one previously found by temporal stability analyses. In addition, a transition from convective to absolute instability was found for sufficiently large values of the Reynolds number and swirl strength. ${ }^{14}$

Despite the aforementioned studies, which deal with fully developed swirling flow in a rotating pipe, the different problem of the stability of a swirling flow developing in the entrance region of a pipe at rest has not been investigated yet. In this paper, we address this problem considering a basic flow at the pipe entrance with an uniform axial velocity profile and with an azimuthal velocity profile of the Burger type. The development of the resulting swirling basic flow in the pipe entrance region has been computed in a selfconsistent manner by solving the steady Navier-Stokes equations in the slender approximation using a standard method of lines. Then, the complete set of Navier-Stokes equations are linearized around that basic solution in order to perform a local, nonparallel, spatial stability analysis at different pipe sections, which permits one to determine the critical Reynolds number beyond which convectively unstable perturbations develop. The results show that even a small amount of swirl modifies completely the picture of the flow with respect to the nonswirling case. In particular, the critical Reynolds numbers found for swirling flows are much smaller than those for nonswirling ones. Also the extent of the region where the flow may become unstable is much larger for the swirling case than for the nonswirling one.

The general problem formulation and the numerical scheme are described in Sec. II. Numerical results on the flow stability are given and discussed in Sec. III. Finally, a summary of the results is given in Sec. IV. 


\section{FORMULATION OF THE PROBLEM}

\section{A. The basic vortex}

Let us investigate the steady, axisymmetric developing swirling flow at the entrance region of a cylindrical pipe of radius $R$. The variables have been made dimensionless using $R$ and $R$ Re as radial and axial length scales, $W_{0}$ as characteristic velocity in the axial and azimuthal directions, $\nu / R$ as characteristic velocity in the radial direction, and $\rho W_{0}^{2}$ as characteristic pressure; $W_{0}$ is the characteristic axial velocity at the entrance and $\rho$ and $\nu$ are the density and kinematic viscosity of the fluid, respectively. If the characteristic Reynolds number, $\operatorname{Re}=W_{0} R / \nu$ is large enough, the velocity components $u_{0}, v_{0}, w_{0}$ in cylindrical coordinates $(r, \phi, z)$ and pressure, $p_{0}$, of the basic swirling flow satisfy, up to order of $\operatorname{Re}^{-2}$, the following:

$$
\begin{aligned}
& \frac{1}{r} \frac{\partial}{\partial r}\left(r u_{0}\right)+\frac{\partial w_{0}}{\partial z}=0, \\
& \frac{v_{0}^{2}}{r}=\frac{\partial p_{0}}{\partial r}, \\
& u_{0} \frac{\partial v_{0}}{\partial r}+w_{0} \frac{\partial v_{0}}{\partial z}+\frac{v_{0} u_{0}}{r}=\left[\frac{1}{r} \frac{\partial}{\partial r}\left(r \frac{\partial v_{0}}{\partial r}\right)-\frac{v_{0}}{r^{2}}\right], \\
& u_{0} \frac{\partial w_{0}}{\partial r}+w_{0} \frac{\partial w_{0}}{\partial z}=-\frac{\partial p_{0}}{\partial z}+\left[\frac{1}{r} \frac{\partial}{\partial r}\left(r \frac{\partial w_{0}}{\partial r}\right)\right] .
\end{aligned}
$$

Equations (1)-(4) have been solved subject to the following boundary conditions.

At the axis, $r=0$,

$$
\begin{aligned}
u_{0} & =v_{0} \\
& =\frac{\partial w_{0}}{\partial r}=0 \quad \text { (regularity and symmetric conditions). }
\end{aligned}
$$

At the pipe wall, $r=1$, the impermeability and nonslip conditions read

$$
v_{0}=w_{0}=u_{0}=0 \text {. }
$$

Since we do not have any information about the flow upstream of the initial station, an uniform axial velocity and an azimuthal velocity profile of the Burgers vortex type have been assumed at the pipe entrance $z=0$,

$$
w_{0}=1, \quad v_{0}=\frac{S \delta}{r}\left[1-\exp \left(-(r / \delta)^{2}\right)\right] .
$$

$\delta<1$ is the dimensionless vortex core length and $S$ is a swirl parameter which characterizes the strength of the vortex at the entrance. Conditions (7) are standard in the swirling flow literature and their use is justified in linear stability analyses of developing swirling flows since the conditions of the flow at the pipe entrance should not affect significantly the development of instabilities due to the presence of a swirl.

To solve Eqs. (1)-(4) with boundary conditions (5)-(7), we have used a standard explicit method of lines. For that purpose, we have first eliminated the pressure in Eqs. (1)-(4) by adding the result of taking derivatives with respect to $z$ in

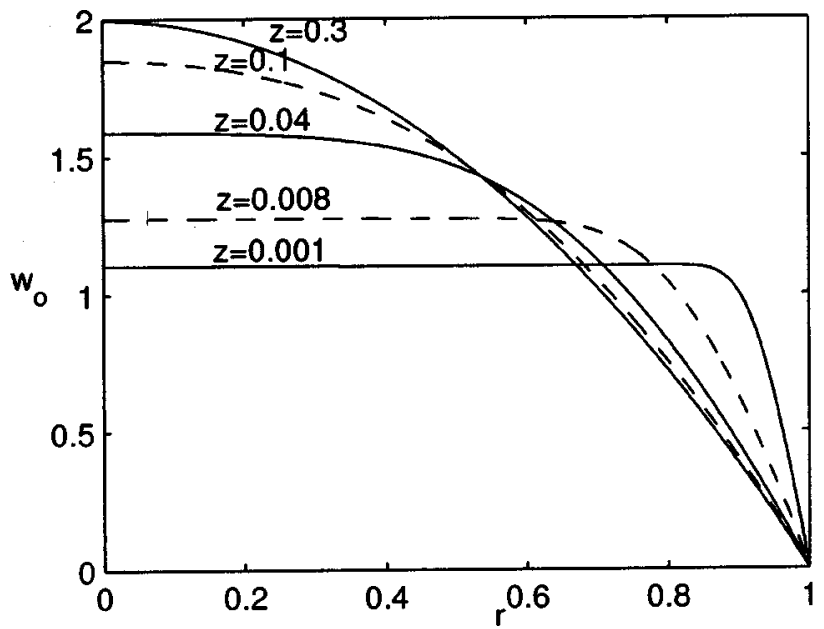

FIG. 1. Radial distribution of the axial velocity of the basic flow at several $z$ stations of the pipe; $S=0.2$ and $\delta=0.5$.

Eq. (2) to that obtained by taking derivatives with respect to $r$ in Eq. (4). The resulting system of equations is then discretized in the radial direction at given points, $r_{i}=(i$ $-1) /(N-1), i=1, \ldots, N$ using second-order central differences, and after some algebra, one can obtain a simple tridiagonal system which yields the values of the radial velocity at the different lines, $\left(u_{0}^{i}\right)_{i=1}^{N}$, in terms of $\left(w_{0}^{i}\right)_{i=1}^{N}$ and $\left(v_{0}^{i}\right)_{i=1}^{N}$. In this manner, one arrives at a system of $2 N$ ordinary differential equations for $(\partial w / \partial z)_{i=1}^{N}$ and $(\partial v / \partial z)_{i=1}^{N}$ which can be solved, for example, with a standard variable step-size fourth-order Runge-Kutta method. In this problem, we have considered $N=400$ lines, and a relative error tolerance of $10^{-6}$ for the Runge-Kutta solver, which allow for a very stable and accurate downstream integration of the equations. It should be remarked that the use of the method of lines avoids complicated and expensive nonlinear iterations required, for example, by implicit finite difference methods.

Figures 1 and 2 show the radial profiles of both the axial velocity (1) and the azimuthal velocity (2) of a basic flow

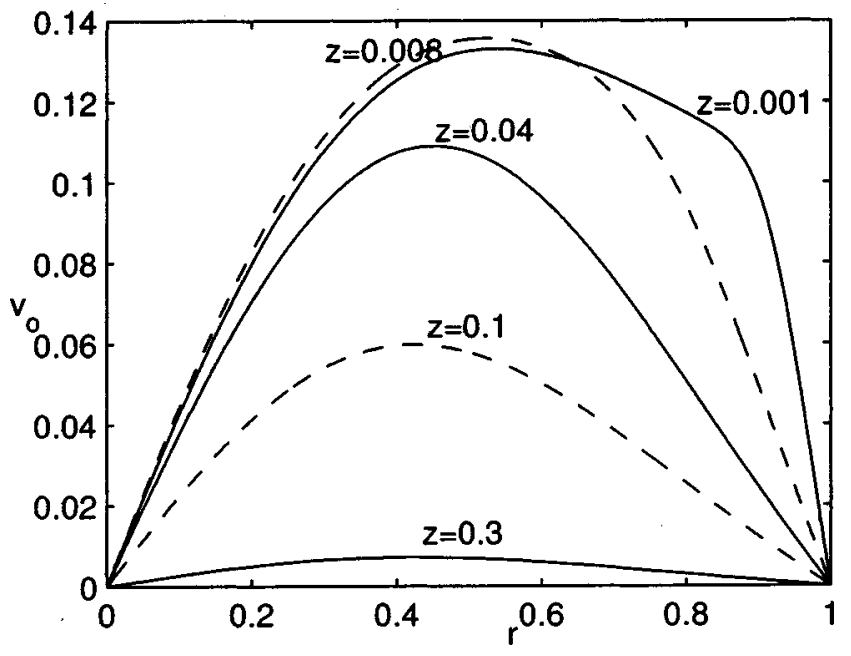

FIG. 2. Radial distribution of the azimuthal velocity of the basic flow at several $z$ stations of the pipe; $S=0.2$ and $\delta=0.5$. 


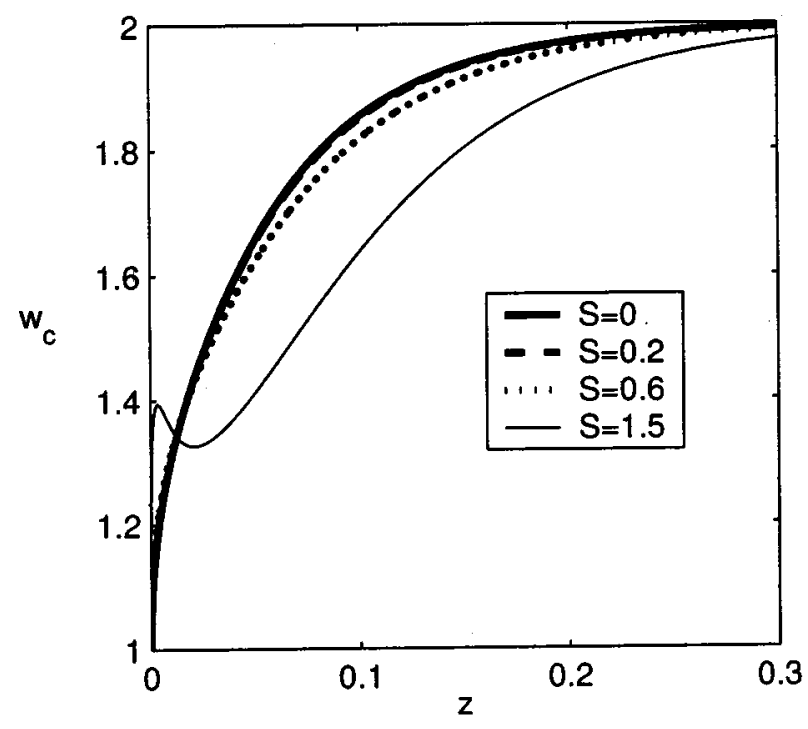

FIG. 3. Velocity at the axis $w_{c}$ as function of $z$ for $\delta=0.5$ and different values of the swirl parameter.

( $S=0.2$ and $\delta=0.5)$ at several $z$ stations of the pipe. Note that the axial velocity evolves downstream toward a profile of the Poiseuille type while the swirl decays.

Figure 3 shows the velocity at the axis, $w_{c} \equiv w(r$ $=0, z)$, as a function of dimensionless pipe axial distance, $z$, for several values of the swirl strength, $S$, and for $\delta=0.5$. It can be observed that in all cases shown in Fig. 3, the classical (nonswirling) Poiseuille flow $\left(w_{c} \rightarrow 2\right)$ is reached sufficiently far from the pipe entrance; the azimuthal velocities being eventually damped by viscous diffusion. For moderately small swirl strengths, the development of the swirling flow does not differ much from the nonswirling one. However for larger swirl strengths $(S=1.5$ in Fig. 3$), w_{c}$ presents a nonmonotonic evolution along the pipe and the distance required to reach the pure Poiseuille flow increases. This increase in the entrance length may be due to the pressure gradient associated with centrifugal forces which lowers the growth rates of the wall boundary layers. Finally, for a swirl strength $S \sim 1.6$ (not shown in the figure) the downstream integration of the basic flow eventually fails at some $z$ station close to the pipe entrance. Some authors ${ }^{15-18}$ have interpreted this failure of the slender (QC) approximation to describe the basic flow as the occurrence of vortex breakdown. However, in this study, we are not interested in the vortex breakdown phenomenon (which requires azimuthal velocities of the same order as the axial ones), but on the linear stability analysis of a developing slender and axisymmetric swirling flow with moderately low $S$ values.

\section{B. Nonparallel local linear stability formulation}

As usual in stability analysis, we split the velocity and pressure fields $(u, v, w, p)$ into two problems: the basic problem $\left(u_{0}, v_{0}, w_{0}, p_{0}\right)$ and a perturbation one $(\bar{u}, \bar{v}, \bar{w}, \bar{p})$,

$$
u=u_{0} / \operatorname{Re}+\bar{u}, \quad v=v_{0}+\bar{v}, \quad w=w_{0}+\bar{w}, \quad p=p_{0}+\bar{p} .
$$

The presence of the Reynolds number in the term representing the basic radial velocity in Eq. (8) is due to the use of $W_{0}$ in the definition of the nondimensional velocity field $(u, v, w)$.

The perturbation vector $s \equiv[\bar{u}, \bar{v}, \bar{w}, \bar{p}]$ is usually written in the standard form:

$$
\mathbf{s}(r, z, \phi, t)=\mathbf{S}(r) X(z, \phi, t),
$$

where the complex eigenfunctions only depend on the radial coordinate

$$
\mathbf{S}(r) \equiv\left[u_{1}(r), v_{1}(r), w_{1}(r), p_{1}(r)\right],
$$

while the exponential part of the perturbations describes the wave-like nature of the disturbance,

$$
\mathbf{X}(z, \phi, t) \equiv \exp [\operatorname{Re} k z+i(n \phi-\omega t)] .
$$

$\omega \equiv \Omega R / W_{0}$ and $k \equiv R k^{*}$ are the nondimensional frequency and the axial wave number, respectively, and $\Omega$ and $k^{*}$ are the corresponding dimensional values. The real and the imaginary parts $\gamma$ and $\alpha$ of the complex axial wave number

$$
k \equiv \gamma+i \alpha,
$$

are the exponential growth rate and the axial wave number, respectively.

Introducing (8) into the Navier-Stokes equations and neglecting terms of order $\mathrm{Re}^{-2}$ and higher, one arrives at the following set of nonparallel, linear, stability equations:

$w_{1} k+\frac{\partial u_{1}}{\partial r}+\frac{u_{1}}{r}+\frac{i n v_{1}}{r}=0$,

$$
\begin{aligned}
i \omega u_{1}-k w_{0} u_{1} & -\frac{u_{0}}{\operatorname{Re}} \frac{\partial u_{1}}{\partial r}-\frac{u_{1}}{\operatorname{Re}} \frac{\partial u_{0}}{\partial r}+\frac{2 v_{0} v_{1}}{r}-\frac{i n v_{0} v_{1}}{r} \\
- & \frac{\partial p_{1}}{\partial r}+\frac{1}{\operatorname{Re}}\left[\frac{\partial^{2} u_{1}}{\partial r^{2}}+\frac{1}{r} \frac{\partial u_{1}}{\partial r}-\left(-k^{2}+\frac{n^{2}}{r^{2}}\right) u_{1}-\frac{u_{1}}{r^{2}}\right. \\
\left.-2 i n \frac{v_{1}}{r^{2}}\right] & =0,
\end{aligned}
$$

$$
\begin{gathered}
i \omega v_{1}-k w_{0} v_{1}-\frac{u_{0}}{\operatorname{Re}} \frac{\partial v_{1}}{\partial r}-\frac{w_{1}}{\operatorname{Re}} \frac{\partial v_{0}}{\partial z}-\frac{u_{0}}{\operatorname{Re}} \frac{v_{1}}{r}-i n \frac{v_{0} v_{1}}{r} \\
-u_{1}\left(\frac{v_{0}}{r}+\frac{\partial v_{0}}{\partial r}\right)-i n \frac{p_{1}}{r}+\frac{1}{\operatorname{Re}}\left[\frac{\partial^{2} v_{1}}{\partial r^{2}}+\frac{1}{r} \frac{\partial v_{1}}{\partial r}\right. \\
\left.-\left(-k^{2}+\frac{n^{2}}{r^{2}}\right) v_{1}+2 i n \frac{u_{1}}{r^{2}}-\frac{v_{1}}{r^{2}}\right]=0,
\end{gathered}
$$

$$
\begin{gathered}
i \omega w_{1}-k w_{0} w_{1}-\frac{u_{0}}{\operatorname{Re}} \frac{\partial w_{1}}{\partial r}-\frac{w_{1}}{\operatorname{Re}} \frac{\partial w_{0}}{\partial z}-u_{1} \frac{\partial w_{0}}{\partial r}-i n \frac{v_{0} w_{1}}{r} \\
-k p_{1}+\frac{1}{\operatorname{Re}}\left[\frac{\partial^{2} w_{1}}{\partial r^{2}}+\frac{1}{r} \frac{\partial w_{1}}{\partial r}-\left(-k^{2}+\frac{n^{2}}{r^{2}}\right) w_{1}\right]=0 .
\end{gathered}
$$

Equations (13)-(16) must be solved subject to the following radial boundary conditions: ${ }^{19}$

$r=1: \quad u_{1}=v_{1}=w_{1}=0 ;$ 


$$
r=0:\left\{\begin{array}{l}
u_{1}=v_{1}=0, \quad \partial w_{1} / \partial r=0, \quad(n=0), \\
u_{1} \pm i v_{1}=0, \quad \partial u_{1} / \partial r=0, \quad w_{1}=0, \quad(n= \pm 1), \\
u_{1}=v_{1}=w_{1}=0, \quad(|n|>1) .
\end{array}\right.
$$

\section{Numerical scheme}

It proves convenient to rewrite Eqs. (13)-(16) in the form

$$
0=\left[\mathbf{L}_{1}+\frac{1}{\operatorname{Re}} \mathbf{L}_{2}+k \mathbf{L}_{3}+\frac{k^{2}}{\operatorname{Re}} \mathbf{L}_{4}\right] \mathbf{S},
$$

where $\mathbf{L}_{1}, \mathbf{L}_{2}, \mathbf{L}_{3}$, and $\mathbf{L}_{4}$ are complex matrices which depend on $z$ and $r$. To solve (19) numerically, the equations are discretized in the $r$ direction using a staggered Chebyshev spectral collocation technique. ${ }^{20}$ Note that no boundary conditions for pressure are needed when using staggered collocation points for pressure.

Let us map the interval $0 \leqslant r \leqslant 1$ into the Chebyshev polynomial domain $-1 \leqslant \xi \leqslant 1$ using the algebraic transformation

$$
r=\frac{1}{2}(1+\xi) .
$$

Then, the $\xi$ coordinate is discretized in $N$ collocation points. $N$, which coincides with the number of Chebyshev polynomials contained in the spectral representation of $\mathbf{S}$, ranges here between 40 and 50 .

The system is solved using the linear companion matrix method described in Ref. 21. The resulting linear eigenvalue problem is solved using an eigenvalue solver subroutine DGVCCG from the IMSL library, which provides the entire spectrum of eigenvalues and eigenfunctions. Spurious eigenvalues were ruled out by comparing the computed spectrums obtained for different values of the number $N$ of collocation points.

\section{RESULTS}

Previously to study the effect of the swirl on the stability of the flow at the entrance region of the pipe, we have analyzed the nonswirling case, $S=0$. In this case, which has been widely investigated in the past, ${ }^{1-5}$ there is only a relevant physical mode (we call this mode the shear mode) that if the Reynolds number is sufficiently high becomes convectively unstable $\left(\gamma>0\right.$ and $\left.c_{g}=\partial \omega / \partial \alpha>0\right),{ }^{22}$ within a certain region close to the pipe inlet. The critical Reynolds number, $\mathrm{Re}^{*}$, at each $z$ station is defined as the minimum Reynolds number for which the shear mode becomes neutrally stable $(\gamma=0)$ at a certain frequency $\omega^{*}$ (critical frequency), while it remains stable $(\gamma<0)$ for any other frequency. The variation of the critical Reynolds number with $z$ for both axisymmetric $n=0$ and nonaxisymmetric perturbation $n=1$ is plotted in Fig. 4. The corresponding variation of the critical frequency $\omega^{*}$ with $z$ is plotted in Fig. 5. Note that, for the nonswirling case considered in these figures, the axisymmetric mode is the most unstable one (absolute minimum value of $\mathrm{Re}^{*}$ ). Also, for Reynolds numbers larger than the minimum value of $\mathrm{Re}^{*}$, the region where the flow is unstable is very close to the pipe entrance; observe that the dimensionless length of

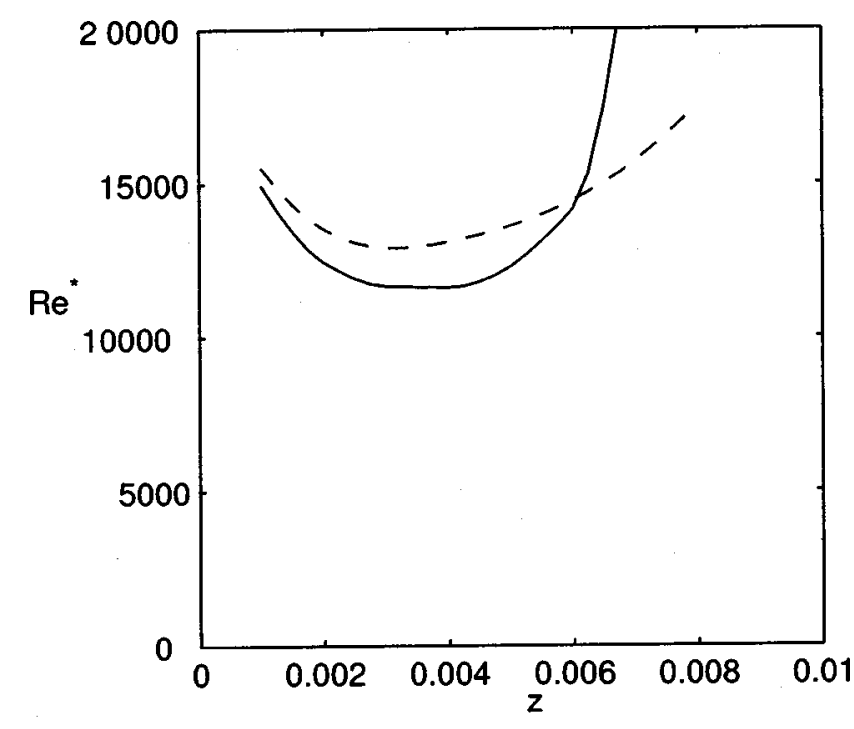

FIG. 4. Critical Reynolds number for nonswirling flows as a function of $z$ : axisymmetric $n=0$ mode (broken line); nonaxisymmetric $n=1$ mode (solid line).

the region is less than 0.01 while the entrance length of the basic flow is roughly thirty times larger, see Fig. 3. These results are in agreement with those obtained in previous linear stability analyses. It should also be pointed out that our numerical results agree pretty well with those obtained by Garg, who also used a nonparallel approximation to describe the stability of the flow. ${ }^{2}$

The stability results get completely modified when flows with a small amount of swirl are considered, even though the downstream evolution of some quantities of the basic flow such as the velocity at the axis (see Fig. 3), the pressure, and the stress at the wall pipe are nearly the same in nonswirling and swirling flows. This is the case of a flow with $S=0.2$ and $\delta=0.5$ for which our numerical results showed the existence of new modes (centrifugal modes) in addition to the shear

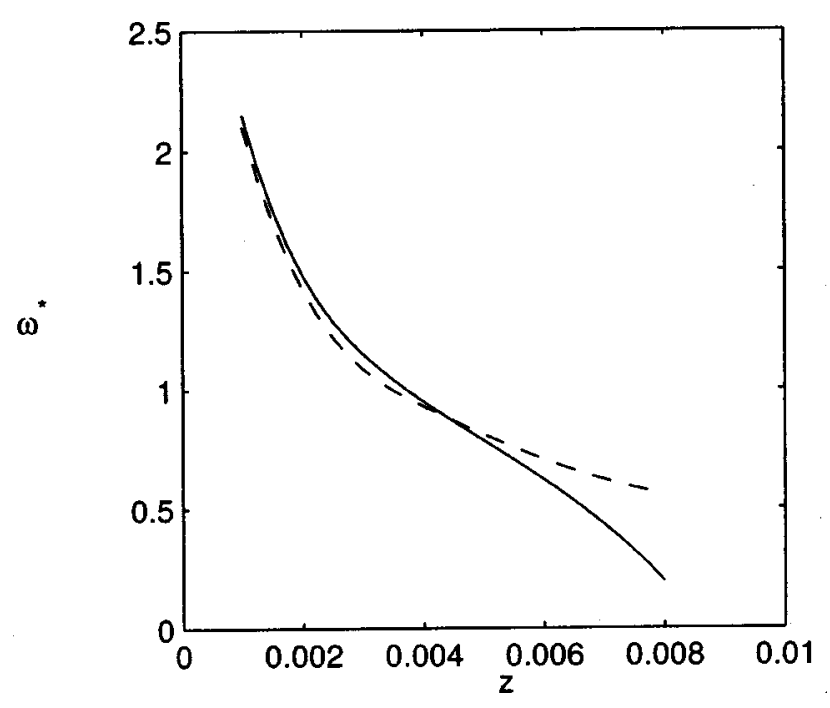

FIG. 5. Critical frequency for nonswirling flows as a function of $z$ : axisymmetric $n=0$ mode (broken line); nonaxisymmetric $n=1$ mode (solid line). 


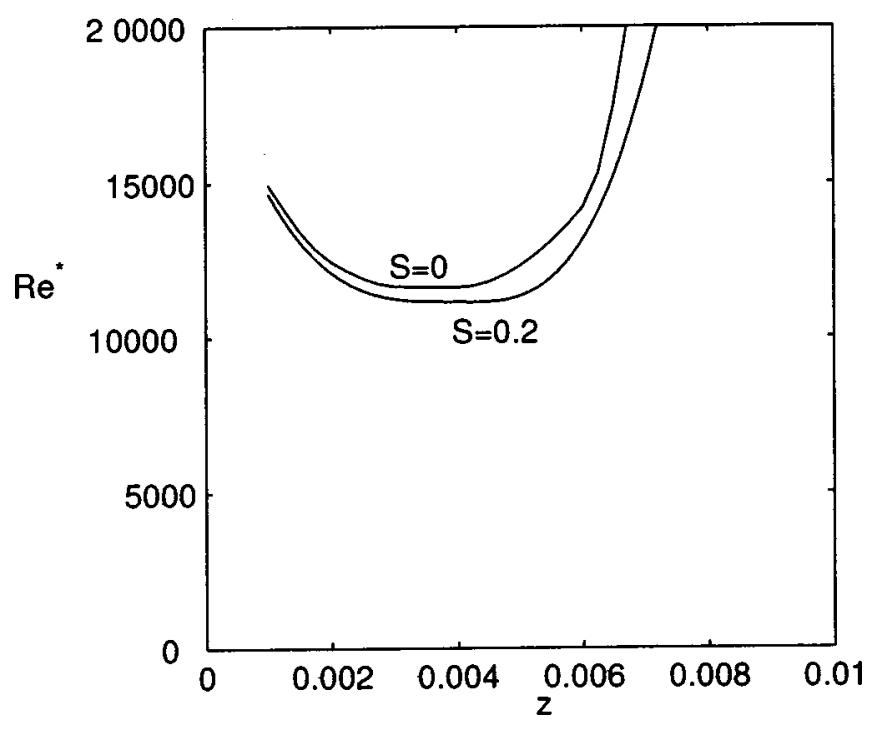

FIG. 6. Critical Reynolds numbers for nonswirling, $S=0$, and swirling, $S$ $=0.2$, flows for axisymmetric $n=0$ modes and $\delta=0.5$.

modes found in the $S=0$ case. The shear modes become convectively unstable for very high Reynolds number (Re 14000) while the centrifugal ones become convectively unstable at much smaller Reynolds numbers. In addition, we have found that the counter-rotating perturbations $(n<0)$ become convectively unstable at Reynolds numbers of the order of 200. Let us point out that centrifugal instabilities at relatively low Reynolds numbers have also been found in the stability analyses of other different swirling flows such as columnar free vortex flows of the Burgers type. $^{23}$

To investigate the appearance of these modes, we have first studied the behavior of the swirling basic flow under axisymmetric perturbations $n=0$. In this case, only shear modes are found, which become unstable at values of $\mathrm{Re}$ slightly smaller than those found in the nonswirling case, see Fig. 6. Observe that the stability characteristics of the axisymmetric modes do not change significantly by the presence of a small amount of swirl in the basic flow. The situation looks quite similar for the case of co-rotating perturbations $(n>0)$, see Fig. 7 ; therefore, we conclude that both axisymmetric and co-rotating modes are of the shear type. A completely different situation presents itself when counter-rotating perturbations $(n<0)$ are considered. In effect, in addition to the shear modes, we have found in this case a new set of modes, which we have called centrifugal modes. These modes become unstable at much smaller Reynolds numbers than those of the shear type. Figure 8 shows the variation of the critical Reynolds number with $z$ for centrifugal modes with azimuthal wave numbers $n=-1, n$ $=-2$, and $n=-3$, respectively. Note in Fig. 8 that the critical Reynolds numbers are not only much smaller than those in the case of shear modes but the extent of the instability region is of the order of the entrance length of the pipe. For example, for $\mathrm{Re}=250$ the flow is unstable under counterrotating perturbations with $n=-1$ in a large portion $(z$ $\sim 0.15$ ) of the entrance region, which extends up to $z \sim 0.3$. In addition, it can be observed in Fig. 8 that the highest order

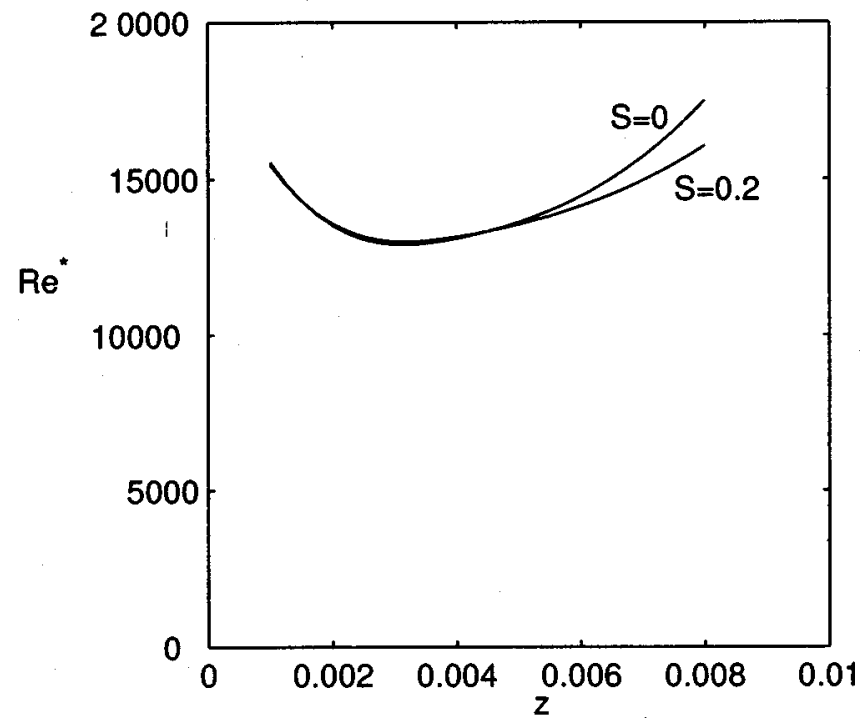

FIG. 7. Critical Reynolds numbers for nonswirling, $S=0$, and swirling, $S$ $=0.2$, flows for co-rotating $n=1$ modes and $\delta=0.5$.

counter-rotating modes are the most unstable ones in a region very close to the pipe entrance, but this tendency quickly changes as we move downstream where the lowest order counter-rotating mode $n=-1$ has the smallest critical Reynolds numbers in most of the entrance region. Therefore, $n=-1$ seems to be the most dangerous mode for the growth of centrifugal instabilities in developing swirling flows. It is worth noting that in the case of a Poiseuille flow with superimposed solid body rotation, the $n=-1$ modes were also the most unstable ones.

Let us now discuss the effect on the stability analysis of $\delta$, which measures the characteristic radius of the vortex core at the pipe entrance [see Eq. (7)]. Thus, a small value of $\delta$ corresponds to the case of a very concentrated near-axis vortex flow, while a value $\delta \sim 1$ corresponds to the case of a swirling flow at the pipe entrance with nearly solid body

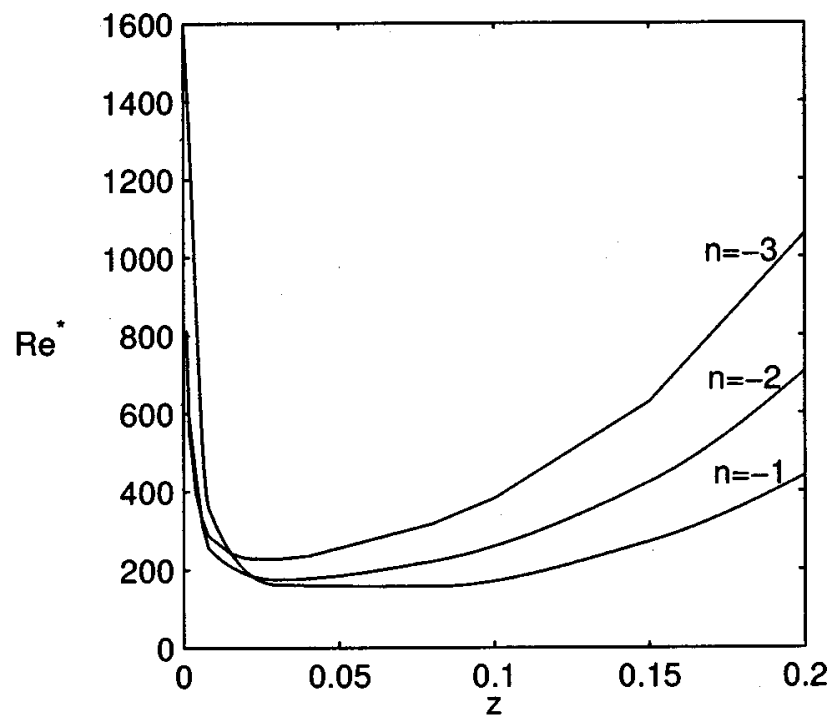

FIG. 8. Critical Reynolds number of the centrifugal modes $n<0$ (counterrotating modes) for $S=0.2$ and $\delta=0.5$. 


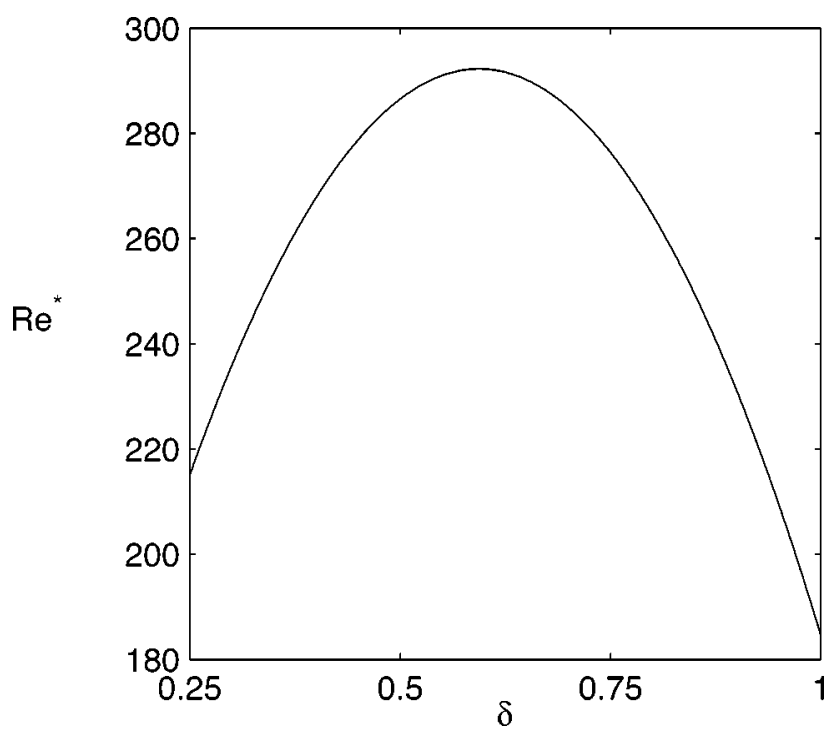

FIG. 9. Critical Reynolds numbers calculated at the pipe section $z=0.08$ as function of $\delta$ for $n=-1$ modes and a swirl strength $S=0.2$.

rotation. For the case $n=-1$, Fig. 9 shows the critical Reynolds number as a function of $\delta$ calculated at the pipe section $z=0.08$, which is the section at which the minimum critical Reynolds number is reached, see Fig. 8. Observe that the evolution of critical Reynolds number with $\delta$ is nonmonotonic, it first increases and then decreases up to reaching its minimum value for the case of almost solid body rotation flows $(\delta=1)$; nevertheless, independent of the concentration of vortex, the flow become unstable for Reynolds numbers larger than 290.

Finally, we have also investigated the effect of the swirl strength $S$ on the flow stability. Again, we have found that the smallest critical Reynolds numbers are obtained for mode $n=-1$ in a pipe section near $z=0.08$. Figure 10 shows the critical Reynolds number as a function of $S$ at $z=0.08$ for

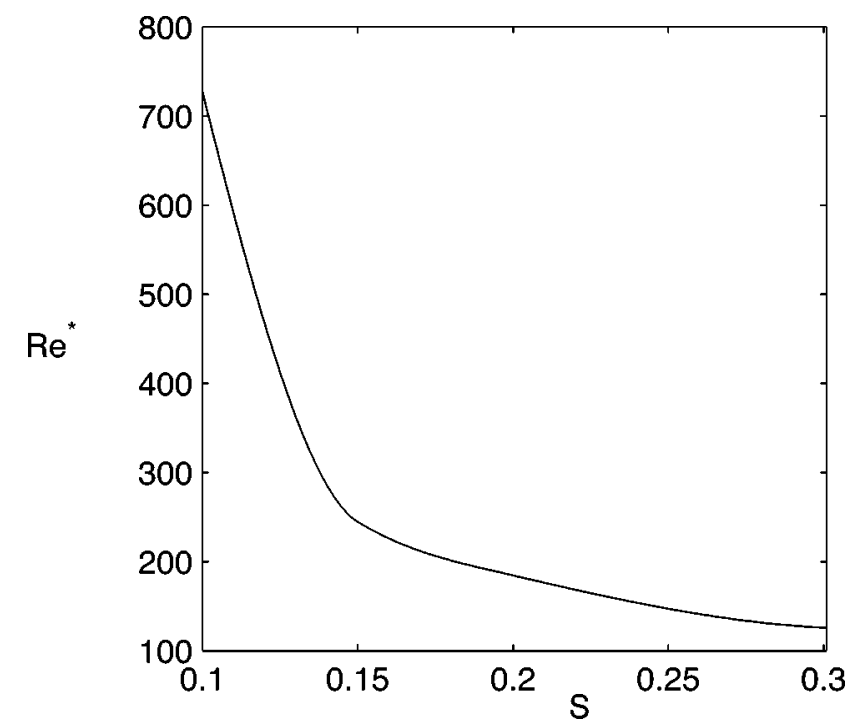

FIG. 10. Critical Reynolds numbers calculated at the pipe section $z=0.08$ as function of $S$ for $n=-1$ modes and $\delta=1$ (solid body rotation at the entrance). $n=-1$ and $\delta=1$. Observe that $\mathrm{Re}^{*}$ decreases monotonically with $S$, but the dependence is much stronger for small $S$ values, for which $\mathrm{Re}^{*}$ becomes very high, in agreement with the results obtained for the case of nonswirling flows.

\section{SUMMARY AND CONCLUSIONS}

We have carried out a spatial, local, viscous, linear stability analysis of developing swirl flows in the entrance region of a cylindrical pipe at rest. The numerical results for the nonswirling case $(S=0)$ confirm the results of previous investigations. Only flows at very large Reynolds numbers develop axisymmetric and nonaxisymmetric shear layer instabilities in a region of the pipe which is located very close to the pipe entrance and whose extent is short compared to the total entrance length. For swirling flows, even at small values of $S$, the stability results are quite different. In this case, we have found both axisymmetric $n=0$ and co-rotating $n>0$ perturbations, which behave much in the same way as the shear layer modes of nonswirling case. On the other hand, a new type of unstable modes associated with counterrotating perturbations $n<0$ appear. These modes, which we have called centrifugal modes, become unstable at much smaller Reynolds numbers and, contrary to the shear modes, the region of flow instability extends to a large portion of the entrance region. Numerical results show that counter-rotating mode $n=-1$ is the most unstable one. It has also been found that even for swirl strength that is not too large, $S=0.2$, the critical Reynolds number for which linear analysis predicts growth of convective instabilities is for the centrifugal modes about 100 times smaller than for the shear ones.

\section{ACKNOWLEDGMENT}

This work has been partially supported by the Directión General de Enseñanza Superior of Spain, Project No. BMF2000-0528.

${ }^{1}$ T. Tatsumi, "Stability of the laminar inlet-flow prior to the formation of Poiseuille Regime. I and II," J. Phys. Soc. Jpn. 7, 489 (1958).

${ }^{2}$ V. K. Garg, "Stability of the developing pipe flow subjected to nonaxisymmetric disturbances," J. Fluid Mech. 110, 209 (1981).

${ }^{3}$ S. C. Gupta and V. K. Garg, "Effect of velocity distribution on the stability of developing flow in a pipe," Phys. Fluids 24, 576 (1981).

${ }^{4}$ L. M. Huang and T. S. Chen, "Stability of developing flow in a pipe: Non-axisymmetric disturbances," Phys. Fluids 17, 245 (1974).

${ }^{5}$ F. Silva and E. A. Moss, "The stability of pipe entrance flows subjected to axisymmetric disturbances," J. Fluids Eng. 116, 61 (1994).

${ }^{6} \mathrm{~T}$. Sarpkaya, "A note on the stability of developing laminar pipe flow subjected to axisymmetric and non-axisymmetric disturbances," J. Fluid Mech. 68, 345 (1975).

${ }^{7}$ T. J. Pedley, "On the instability of rapidly rotating shear flows to nonaxisymmetric disturbances," J. Fluid Mech. 31, 603 (1968).

${ }^{8}$ T. J. Pedley, "On the instability of viscous flow in a rapidly rotating pipe," J. Fluid Mech. 35, 97 (1969).

${ }^{9}$ S. A. Maslowe, "Instability of rigidly rotating flows to non-axisymmetric disturbances," J. Fluid Mech. 64, 307 (1974).

${ }^{10}$ P. A. Mackrodt, "Stability of Hagen-Poiseuille flow with superimposed rigid rotation," J. Fluid Mech. 73, 153 (1976).

${ }^{11}$ F. W. Cotton and H. Salwen, "Linear stability of rotating HagenPoiseuille flow," J. Fluid Mech. 108, 101 (1981).

${ }^{12} \mathrm{~S}$. A. Maslowe and K. Stewarson, "On the linear inviscid stability of rotating Poiseuille flow,” Phys. Fluids 25, 1517 (1982).

${ }^{13}$ M. R. Khorrami, M. R. Malik, and R. L. Ash, "Application of spectral 
collocation techniques to the stability of swirl flows," J. Comput. Phys. 81, 206 (1989).

${ }^{14}$ R. Fernández-Feria and C. del Pino, "The onset of absolute instability of rotating Hagen-Poiseuille flow: A spatial stability analysis," Phys. Fluids 14, 3087 (2002).

${ }^{15}$ M. G. Hall, "Vortex breakdown,” Annu. Rev. Fluid Mech. 4, 195 (1972).

${ }^{16}$ R. Fernández-Feria, J. Fernández de la Mora, and A. Barrero, "Solution breakdown in a family of self-similar nearly inviscid axisymmetric vortices," J. Fluid Mech. 305, 77 (1995).

${ }^{17}$ M. Pérez-Saborid, M. A. Herrada, A. Gómez-Barea, and A. Barrero, "Down-stream evolution of unconfined vortices: Mechanical and thermal aspects," J. Fluid Mech. 471, 51 (2002).

${ }^{18}$ M. A. Herrada, M. Pérez-Saborid, and A. Barrero, "Vortex breakdown in compressible flows in pipes," Phys. Fluids 15, 2208 (2003).

${ }^{19} \mathrm{G}$. K. Batchelor and A. E. Gill, "Analysis of the stability of axisymmetric jets," J. Fluid Mech. 14, 529 (1962).

${ }^{20}$ M. R. Khorrami, "A Chebychev spectral collocation method using a staggered grid for the stability of cylindrical flows," Int. J. Numer. Methods Fluids 12, 825 (1991).

${ }^{21}$ T. J. Bridges and P. J. Morris, "Differential eigenvalue problems in which the parameter appears nonlinearly," J. Comput. Phys. 55, 437 (1984).

${ }^{22}$ P. Huerre and P. A. Monkewitz, "Local and global instabilities in spatially developing flows," Annu. Rev. Fluid Mech. 22, 473 (1990).

${ }^{23}$ E. W. Meyer and K. G. Powell, "Viscous and inviscid instabilities of a trailing vortex," J. Fluid Mech. 245, 91 (1992). 